\title{
Dietary fish oil reduces glomerular injury and elevated renal hydroxyeicosatetraenoic acid levels in the JCR:LA-cp rat, a model of the metabolic syndrome
}

\author{
Harold M. Aukema ${ }^{1 *}$, Jing $\mathrm{Lu}^{2}$, Faye Borthwick ${ }^{2}$ and Spencer D. Proctor ${ }^{2}$ \\ ${ }^{1}$ Department of Human Nutritional Sciences, University of Manitoba, Winnipeg, Manitoba, Canada \\ ${ }^{2}$ Metabolic and Cardiovascular Diseases Laboratory, Alberta Diabetes and Mazankowski Heart Institutes, University of \\ Alberta, Edmonton, Alberta, Canada \\ (Submitted 2 April 2012 - Final revision received 5 September 2012 - Accepted 5 September 2012 - First published online 15 November 2012)
}

\section{Abstract}

We have previously shown nutritional intervention with fish oil ( $n$-3 PUFA) to reduce numerous complications associated with the metabolic syndrome (MetS) in the JCR:LA-corpulent ( $c p)$ rat. In the present study, we sought to explore the potential role of fish oil to prevent glomerulosclerosis in JCR:LA- $c$ p rats via renal eicosanoid metabolism and lipidomic analysis. Male lean and MetS JCR:LA- $c p$ rats were fed a lipid-balanced diet supplemented with fish oil ( 5 or $10 \%$ of total fat). After 16 weeks of feeding, albuminuria was significantly reduced in MetS rats supplemented with 5 or $10 \%$ fish oil ( -53 and $-70 \%$, respectively, compared with the untreated MetS rats). The $5 \%$ fish oil diet resulted in markedly lower glomerulosclerosis (-43\%) in MetS rats and to a lesser extent in those supplemented with $10 \%$ fish oil. Interestingly, untreated MetS rats had higher levels of 11- and 12-hydroxyeicosatetraenoic acids (HETE) $v$. lean rats. Dietary fish oil reduced these levels, as well as other (5-, 9- and 15-) HETE. Whilst genotype did not alter prostanoid levels, fish oil reduced endogenous renal levels of 6-keto $\mathrm{PGF}_{1 \alpha}\left(\mathrm{PGI}_{2}\right.$ metabolite), thromboxane $\mathrm{B}_{2}\left(\mathrm{TxB}_{2}\right), \mathrm{PGF}_{2 \alpha}$ and $\mathrm{PGD}_{2}$ by approximately $60 \%$ in rats fed $10 \%$ fish oil, and $\mathrm{TxB}_{2}(-50 \%)$ and $\mathrm{PGF}_{2 \alpha}(-41 \%)$ in rats fed $5 \%$ fish oil. In conclusion, dietary fish oil prevented glomerular damage in MetS rats and mitigated the elevation in renal HETE levels. These results suggest a potential role for dietary fish oil to improve dysfunctional renal eicosanoid metabolism associated with kidney damage during conditions of the MetS.

Key words: Metabolic syndrome: Renal disease: Eicosanoids: Fish oil (n-3 PUFA)

Long-term sequelae of the metabolic syndrome (MetS) include the clinical presentation of both macro- and microvascular disease $^{(1)}$. The obese, insulin-resistant JCR:LA-corpulent $(c p)$ rat, homozygous for the corpulent trait $(c p / c p)$, is a unique model of the $\mathrm{MetS}^{(1-4)}$. This model displays characteristic macro- and microvascular complications of the MetS, including effects on the kidney such as glomerulosclerosis, proteinuria, decline in renal function and ultimately end-stage renal disease, consistent with human pathological complications $^{(5)}$. Indeed, all of the diagnostic criteria of the MetS are associated with reduced renal function and elevated proteinuria and albuminuria ${ }^{(6)}$. Further, circulating insulin and lipids are contributing factors to the detrimental effects of the MetS on renal function, affecting the glomerulus, causing damage during obesity and diabetes, resulting in glomerulosclerosis and proteinuria even in the early stages of these disorders $^{(7-11)}$.
We recently reported that MetS JCR:LA-cp rats treated with dietary fish oil (rich in $n$-3 PUFA) showed improvements in fasting and postprandial dyslipidaemia, hyperinsulinaemia and the frequency of late-stage myocardial lesions ${ }^{(12)}$. Consequently, we hypothesised that improvements to hyperlipidaemia and hyperinsulinaemia by dietary fish oil may also have beneficial renal effects in the JCR:LA- $c$ p rat, potentially via alterations to eicosanoid metabolism.

Eicosanoids are primarily produced from arachidonic acid (ARA) by cyclo-oxygenase (COX) enzymes, yielding prostanoids, and by lipoxygenase (LOX) enzymes to produce leukotrienes and hydroxy fatty acids (hydroxyeicosatetraenoic acid, HETE). Prostanoids, such as $\mathrm{PGE}_{2}$ and thromboxane $\mathrm{A}_{2}$, derived via the COX pathway, regulate glomerular filtration rate, water and salt homeostasis, as well as inflammatory and fibrotic processes in response to kidney damage ${ }^{(13-15)}$. In diabetic kidneys, increased COX or prostanoid levels are

Abbreviations: ARA, arachidonic acid; COX, cyclo-oxygenase; $c p$, corpulent; HETE, hydroxyeicosatetraenoic acid; LBD, lipid-balanced diet; LOX, lipoxygenase; MetS, metabolic syndrome; TxB2, thromboxane $\mathrm{B}_{2}$.

*Corresponding author: H. M. Aukema, fax +1 204237 4018, email aukema@umanitoba.ca 
associated with development of the disease, and inhibition of this enzyme results in nephroprotection ${ }^{(16-20)}$.

Renal 12-HETE is a product of the $12 / 15$ LOX enzyme and is a vasoconstrictor in the kidney ${ }^{(20)}$, which also has been shown to be associated with experimental diabetic nephropathy ${ }^{(21-23)}$. Pharmacological inhibition or gene knockout of 12/15 LOX reduces glomerular volume, mesangial cell hypertrophy, extracellular matrix formation and proteinuria ${ }^{(24-26)}$. Such effects of select eicosanoids in diabetic nephropathy suggest a role in renal injury during the MetS. Due to the multiple interactions and roles of select eicosanoids in the kidney ${ }^{(20,27)}$, understanding changes in both prostanoids and HETE may reveal mechanisms underlying renal injury during the MetS.

Fish oil ( $n$-3 PUFA) can both compete with ARA for eicosanoid synthetic enzymes as well as inhibit these enzymes. Furthermore, eicosanoids derived from $n$ - 3 PUFA are generally less potent than those derived from ARA ${ }^{(28-30)}$. Supplementation with $n$-3 PUFA provides nephroprotection in a variety of models of renal disease ${ }^{(31-37)}$. Hence, the objectives of the present study were to determine the long-term renal effects of fish oil feeding in a model of the MetS, with a particular focus on glomerular injury and renal eicosanoids.

\section{Materials and methods}

\section{Animal model and experimental procedures}

Male JCR:LA- $c p$ rats, both with the MetS $(c p / c p)$ and lean $(+/$ ?), were raised in an established breeding colony at the University of Alberta, as previously described ${ }^{(3)}$. Rats were weaned at 6 weeks, and allowed to age until week 8. MetS rats were randomly allocated to one of three diets (Table 1); a control hypercholesterolaemic isoenergetic lipid-balanced diet (LBD; $n$ 8); a LBD supplemented with $5 \%(n 8)$ or $10 \%$ ( $n$ 8) fish oil (Table 1$)$ for 16 weeks. Lean rats $(n 8)$, of the same age, were fed the control LBD diet (Table 1) for 16 weeks. Food consumption and body weight were recorded throughout the study. At 24 weeks of age, rats were fasted overnight and killed under isoflurane anaesthesia. Liver, kidney and peri-renal fat pads were weighed and snapfrozen in liquid $\mathrm{N}_{2}$ at $-80^{\circ} \mathrm{C}$, for subsequent analysis where appropriate. Animal care and experimental procedures were conducted in accordance with the Canadian Council on Animal Care and approved by the University of Alberta Animal Care and Use Committee (ACUC-Livestock).

\section{Urine samples}

Following 16 weeks of feeding, rats were euthanised in the fasted state and urine samples were collected. Urine albumin and creatinine concentrations were measured using immunoturbidimetric and Jaffé methods, respectively ${ }^{(5,38)}$. The laboratory CV for albuminuria test was 8.0 (level 1) and $3.2 \%$ (level 2).

\section{Renal histology}

After killing, the left kidney from all animals was excised and fixed in formalin. After conventional processing, kidney sections were examined to quantify glomerulosclerosis, as per the method of Schäfer ${ }^{(39)}$. In brief, each kidney was divided along the long axis, fixed, sectioned and stained with haematoxylin and $\operatorname{cosin}^{(5)}$. For each kidney, ten random fields were viewed ( $\times 4$ objective) and recorded digitally. A total of eight

Table 1. Nutrient and lipid summaries for each dietary group*

\begin{tabular}{|c|c|c|c|}
\hline & Control diet (LBD) & $5 \%$ FO diet† & $10 \%$ FO dietł \\
\hline \multicolumn{4}{|l|}{ Nutrient summary (per kg) } \\
\hline Casein $(\mathrm{g})$ & 270 & 270 & 270 \\
\hline Starch (g) & 214 & 214 & 214 \\
\hline Dextrose $(\mathrm{g})$ & 217 & 217 & 217 \\
\hline Non-nutritive cellulose (g) & 80 & 80 & 80 \\
\hline Vitamin mixture $(\mathrm{g})$ & $9 \cdot 5$ & $9 \cdot 5$ & $9 \cdot 5$ \\
\hline Mineral mix (g) & 48 & 48 & 48 \\
\hline Choline (g) & $2 \cdot 75$ & $2 \cdot 75$ & $2 \cdot 75$ \\
\hline Inositol (g) & $6 \cdot 25$ & $6 \cdot 25$ & $6 \cdot 25$ \\
\hline L-Met (g) & 2.5 & 2.5 & 2.5 \\
\hline Linseed oil (g) & 3.0 & 3.0 & 3.0 \\
\hline Tallow (g) & $91 \cdot 71$ & $91 \cdot 88$ & 94.73 \\
\hline Sunflower oil (g) & $55 \cdot 29$ & $40 \cdot 13$ & $24 \cdot 27$ \\
\hline Fish oil $(\mathrm{g}) \S$ & 0.0 & 15 & 28 \\
\hline Cholesterol (g) & 10 & 10 & 10 \\
\hline \multicolumn{4}{|l|}{ Lipid summary (\% of fat) } \\
\hline Total polyunsaturated fat & $24 \cdot 7$ & $24 \cdot 3$ & $27 \cdot 7$ \\
\hline Total saturated fat & $64 \cdot 5$ & $66 \cdot 0$ & $65 \cdot 0$ \\
\hline Polysaturated:saturated ratio & 0.4 & 0.4 & 0.4 \\
\hline Total $n-6$ & $23 \cdot 4$ & $17 \cdot 4$ & $17 \cdot 1$ \\
\hline Total $n-3$ & $1 \cdot 2$ & 6.9 & $10 \cdot 6$ \\
\hline Total EPA + DHA & 0.0 & $5 \cdot 3$ & $9 \cdot 4$ \\
\hline
\end{tabular}


complete glomeruli in each field of view were blindly scored as sclerotic (mild-to-severe glomerular sclerosis) or normal (minimal sclerosis or normal). The fraction of sclerotic glomeruli was calculated for each kidney and data represented as the average incidence of sclerotic glomeruli in each kidney.

\section{Eicosanoid analysis}

Lyophilised tissue ( $45 \mathrm{mg}$ ) from the right kidney was homogenised on ice in $1.25 \mathrm{ml}$ of freshly prepared Tyrodes buffer $^{(40)}$. After homogenisation, Triton X-100 (Fisher Scientific) was added and mixed to achieve a final concentration of $0.01 \%$. Endogenous eicosanoid levels and in vitro production were determined in aliquots $(200 \mu \mathrm{l})$ under the following conditions: (1) $0 \mathrm{~min}$ for determination of endogenous levels and (2) $10 \mathrm{~min}$ at $37^{\circ} \mathrm{C}$ for determination of in vitro production, as described $^{(40)}$. Reactions were stopped with $1 \%$ formic acid in methanol and eicosanoids extracted in acidified methanolwater-ethanol (5:10:1, by vol.) containing $10 \mu \mathrm{l}$ of antioxidant solution $(0 \cdot 2 \mathrm{mg} / \mathrm{ml}$ butylated hydroxytoluene, $0.2 \mathrm{mg} / \mathrm{ml}$ EDTA, $2 \mathrm{mg} / \mathrm{ml}$ triphenylphosphine, $2 \mathrm{mg} / \mathrm{ml}$ indomethacin in a solution of 2:1:1 methanol-ethanol-water). An internal standard mix consisting of $7 \cdot 5-25 \mathrm{ng}$ of ${ }^{2} \mathrm{H}$-labelled standards (mentioned later) was added. After centrifugation, supernatants $(\mathrm{pH}<3.0)$ were applied to Strata-X SPE columns (Phenomenex) pre-conditioned with methanol and water ( $\mathrm{pH} 3$ ). After loading, columns were washed in $10 \%$ methanol in water ( $\mathrm{pH} 3$ ) and samples eluted with $100 \%$ methanol $^{(41)}$. Liquid chromatography tandem MS (liquid chromatography/ MS/MS) was performed based on the method described by Deems et al. ${ }^{(41)}$. Dried down samples were re-suspended in water-acetonitrile-formic acid (70:30:0.02, by vol., solvent A) and eicosanoids separated by reverse-phase HPLC using a C18 column (Luna, $250 \times 2.0 \mathrm{~mm}$, Phenomenex) at a flow rate of $300 \mu \mathrm{l} / \mathrm{min}$. The column was equilibrated in solvent $\mathrm{A}$ and samples were eluted with a linear gradient from 0 to $20 \%$ solvent B (acetonitrile-isopropyl alcohol, 50:50; v/v) for $11 \mathrm{~min}$, then increased to $100 \%$ by $13 \mathrm{~min}$ and held until $16 \mathrm{~min}$, then dropped to $0 \%$ by $16 \mathrm{~min}$ and held until $19 \mathrm{~min}$. The HPLC was coupled to a triple quadrupole tandem mass spectrometer (API 2000) with electrospray ionisation source (Applied Biosystems). Eicosanoids were analysed via multiple-reaction monitoring in negative ionisation mode. Mass transitions of ${ }^{2} \mathrm{H}$-labelled standards and eicosanoids were as follows: 5 -HETE-d $\mathrm{d}_{8}(\mathrm{~m} / \mathrm{z} 327 \rightarrow 116)$ for 5 -HETE $(\mathrm{m} / z 319 \rightarrow$ $115), 8$-HETE $(m / z 319 \rightarrow 155)$, 9-HETE $(m / z 319 \rightarrow 151)$ and 11 -HETE $(m / z 319 \rightarrow 167)$; 15 -HETE-d $8(m / z 327 \rightarrow 226)$ for 12 -HETE $(m / z \quad 319 \rightarrow 179)$ and 15 -HETE $(m / z \quad 319 \rightarrow 219)$; 6-keto $\mathrm{PGF}_{1 \alpha}-\mathrm{d}_{4}(\mathrm{~m} / z 373 \rightarrow 211)$ for 6-keto $\mathrm{PGF}_{1 \alpha}(\mathrm{m} / \mathrm{z}$ $369 \rightarrow 163)$; thromboxane $\mathrm{B}_{2}\left(\mathrm{TxB}_{2}\right)-\mathrm{d}_{4}(m / z 373 \rightarrow 173)$ for $\mathrm{TxB}_{2}(m / z 369 \rightarrow 169) ; \mathrm{PGF}_{2 \alpha}-\mathrm{d}_{4}(m / z 357 \rightarrow 197)$ for $\mathrm{PGF}_{2 \alpha}$ $(m / z 353 \rightarrow 193) ; \mathrm{PGE}_{2}-\mathrm{d}_{4}(m / z 355 \rightarrow 275)$ for $\mathrm{PGE}_{2}(m / z$ $351 \rightarrow 271)$ and $\mathrm{PGE}_{3}(\mathrm{~m} / z 349 \rightarrow 269) ; \mathrm{PGD}_{2}-\mathrm{d}_{4}(\mathrm{~m} / z 355 \rightarrow$ 193) for $\mathrm{PGD}_{2}(m / z 351 \rightarrow 189)$ and $\mathrm{PGD}_{3}(m / z 349 \rightarrow 269)$. Quantification of eicosanoids was determined using the stable isotope dilution $\operatorname{method}^{(42)}$.

\section{Statistical analysis}

Data were tested for normal distribution and differences between the MetS control ( $c p / c p$ LBD) compared with the lean control $(+/$ ? LBD) group were analysed using the unpaired $t$ test $(P<0 \cdot 05)$. Statistical differences between the MetS fish oil treatment (5 and 10\%) groups compared with the MetS control ( $c p / c p$ LBD) group were analysed by one-way ANOVA followed by Tukey's post hoc tests, with significance set at $P<0.05$ (Sigma Stat, Jandel Scientific and GraphPad Prism, GraphPad Software Inc.). For data that could not be normalised by logarithmic transformation, the Kruskal-Wallis test was used. All results are shown as means with their standard errors.

\section{Results}

\section{Liver, kidney and peri-renal fat pad weight}

Untreated MetS rats had significantly greater liver weight (2-fold; $P<0.05$ ), compared with the lean control rats (Table 2). MetS rats fed a $5 \%$ fish oil diet for 16 weeks had significantly lower liver weight $(-29 \% ; P<0.05)$, compared with the untreated MetS rats (Table 2). Relative to lean control rats, untreated MetS rats had significantly increased kidney weight $(27 \% ; P<0.05)$ (Table 2 ). There was no significant difference in kidney weight of either 5 or $10 \%$ fish oil

Table 2. Body and organ weights of lean and metabolic syndrome (MetS) male JCR:LA-cp rats fed a lipid-balanced control diet (LBD), or a 5 or $10 \%$ fish oil (FO) diet

(Mean values with their standard errors, $n 8$ per group)

\begin{tabular}{|c|c|c|c|c|c|c|c|c|}
\hline \multirow{3}{*}{$\begin{array}{l}\text { Genotype... } \\
\text { Diet... }\end{array}$} & \multirow{2}{*}{\multicolumn{2}{|c|}{$\frac{\text { Control }}{\text { LBD }}$}} & \multirow{2}{*}{\multicolumn{2}{|c|}{$\frac{\text { MetS }}{\text { LBD }}$}} & \multirow{2}{*}{\multicolumn{2}{|c|}{ MetS $+5 \%$ FO }} & \multirow{2}{*}{\multicolumn{2}{|c|}{ MetS $+10 \%$ FO }} \\
\hline & & & & & & & & \\
\hline & Mean & SEM & Mean & SEM & Mean & SEM & Mean & SEM \\
\hline Body weight (g) & 375.4 & $10 \cdot 3$ & 668.5 & $8 \cdot 2^{\star}$ & $584 \cdot 0$ & 11.8 & 552.4 & $10 \cdot 0 \dagger$ \\
\hline Kidney weight (g) & 1.26 & 0.07 & 1.60 & $0.07^{\star}$ & 1.41 & 0.05 & 1.49 & 0.07 \\
\hline Kidney:body weight ratio (g/kg) & 3.4 & 0.2 & $2 \cdot 4$ & $0 \cdot 1^{\star *}$ & $2 \cdot 4$ & 0.3 & $2 \cdot 7$ & 0.2 \\
\hline Liver weight (g) & 9.9 & 0.5 & $20 \cdot 0$ & $0.6^{*}$ & 14.3 & $1.8 \dagger$ & $16 \cdot 3$ & 0.4 \\
\hline Perirenal fat pad weight $(\mathrm{g})$ & 0.94 & 0.45 & 9.99 & $2 \cdot 67^{\star}$ & 6.59 & $1.31+\dagger \dagger$ & 4.25 & $1.06+† \dagger$ \\
\hline Perirenal fat pad weight:body weight ratio $(\mathrm{g} / \mathrm{kg})$ & $2 \cdot 0$ & 1.0 & $15 \cdot 0$ & 3.0 & $11 \cdot 0$ & $2.0 \dagger$ & $8 \cdot 0$ & $2.0+\dagger$ \\
\hline
\end{tabular}

Mean values were significantly different from that of the lean control: ${ }^{*} P<0.05,{ }^{\star *} P<0.01$.

Mean values were significantly different from that of the MetS control: $\dagger P<0.05, \uparrow \dagger P<0.01, \dagger \dagger \dagger P<0.001$. 
groups (Table 2), when compared with the untreated MetS rats. Compared with the lean group, all MetS groups had a higher peri-renal fat pad weight and ratio of peri-renal fat pad weight to body weight (5- to 11-fold increase) (Table 2). However, relative to untreated MetS rats, both 5 and $10 \%$ fish oil-treated rats had significantly lower perirenal fat pad weight $(-34$ to $57 \% ; P<0.001)$ (Table 2$)$ and ratio of peri-renal fat pad weight to body weight $(-27$ to $47 \% ; P<0.01$ ) (Table 2).

\section{Urinary biochemical profile}

Urinary albumin/creatinine concentrations were markedly higher (78.5-fold; $P<0.001)$ in untreated MetS rats compared with the lean control rats (Fig. 1), consistent with previously published work by our group ${ }^{(5)}$. After 16 weeks of feeding, rats fed the fish oil diet ( 5 or $10 \%$ ) had lower urine albumin excretion compared with the untreated MetS rats $(-53$ and $-70 \%$, respectively; $P<0.01$ and $P<0.001$ ) (Fig. 1).

\section{Glomerulosclerosis}

Untreated MetS rats exhibited a substantial increase in the frequency of glomerulosclerosis compared with the lean control rats (6.4-fold; $P<0.001$ ) (Fig. 2) and is consistent with previous studies using this rat model ${ }^{(5)}$. Further, untreated MetS rats exhibited a greater severity of glomerulosclerosis and interstitial inflammation compared with their lean counterparts (Fig. 2). MetS rats treated with $5 \%$ fish oil had a significant reduction in the fraction of sclerotic glomeruli $(-43 \%$; $P<0 \cdot 001$ ), relative to the untreated MetS group (Fig. 2). A similar significance was not achieved in the $10 \%$ fish oil group. Intriguingly, sporadic tubular damage was observed in kidneys of MetS rats fed the $10 \%$ fish oil diet (Fig. 2(b-e)).

\section{Renal eicosanoid levels and in vitro production}

The levels of 11-HETE and 12-HETE were 71 and 109\% higher, respectively, in untreated MetS compared with the

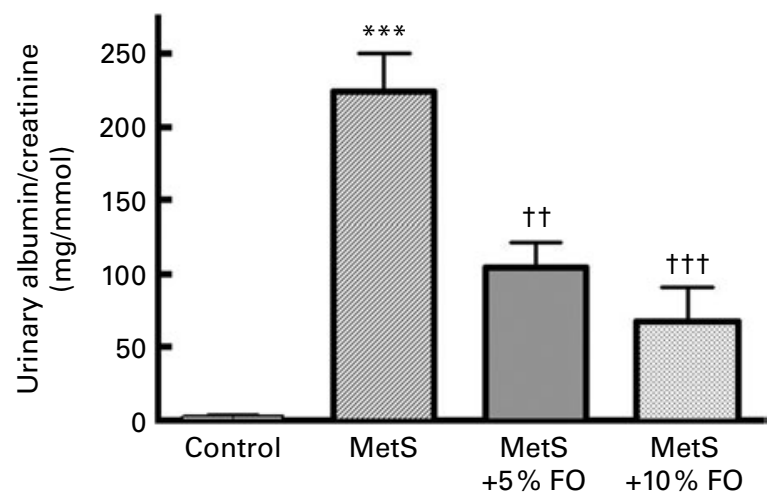

Fig. 1. The ratio of urinary albumin to creatinine levels of JCR:LA- $c p$ rats in each of the treatment groups. Values are means, with standard errors represented by vertical bars $(n 8)$. Mean value was significantly different from that of the lean control: ${ }^{\star \star \star} P<0.001$. Mean value was significantly different from that of the metabolic syndrome (MetS) control: $1+P<0.01$, ††† $P<0.001$. FO, fish oil.

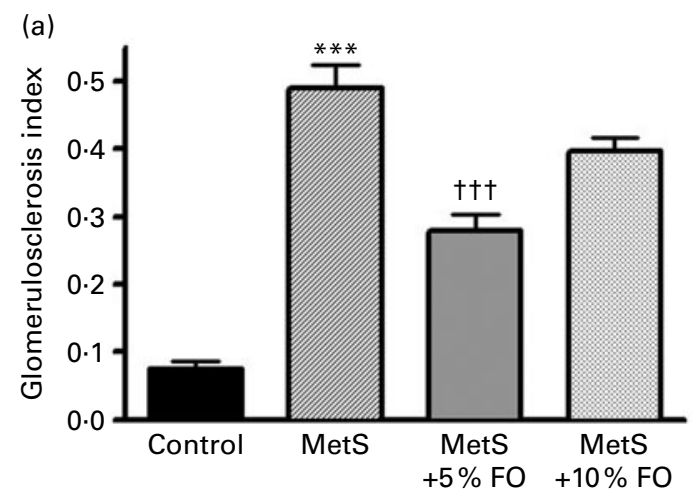

(b)

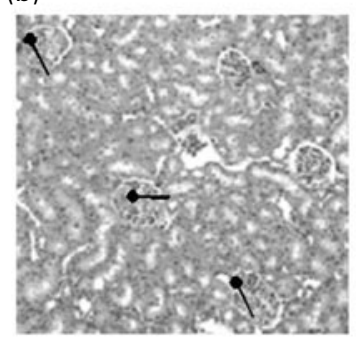

(d)

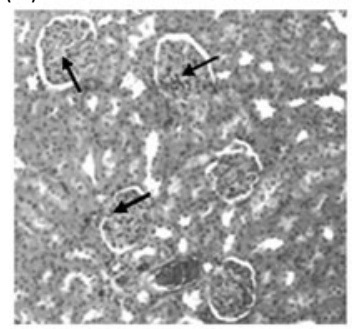

(c)

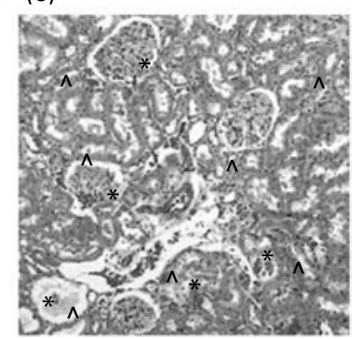

(e)

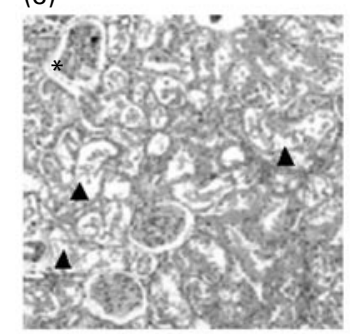

Fig. 2. (a) Proportion of sclerotic glomeruli in JCR:LA-cp rats in each of the treatment groups. Values are means, with standard errors represented by vertical bars $(n 8)$. Mean value was significantly different from that of the lean control: ${ }^{* * *} P<0.001$. Mean value was significantly different from that of the metabolic syndrome (MetS) control: ††† $P<0.001$. (b-e) Kidney sections stained by haematoxylin and eosin. Representative micrographs of kidneys from (b) normal glomeruli $(\bullet)$ of lean control rats. (c) Kidneys of MetS control rats illustrating some sclerotic glomeruli including interstitial inflammation and malformed glomeruli $(\wedge)$. (d) Glomeruli from MetS rats treated with $5 \%$ fish oil (FO) showing relatively normal glomeruli $(\uparrow)$. (e) Glomeruli from MetS rats treated with $10 \%$ FO showing malformed tubular structure $(\mathbf{\Lambda})$ and glomerulosclerosis. The tubule structures were malformed by fat vacuoles.

lean rat kidneys (Fig. 3(d) and (e)). These levels were reduced by fish oil feeding to similar values observed in lean rats fed the control diet. Renal 15-HETE levels followed the same pattern (Fig. 3(f)), with the exception that the difference between MetS and lean rats was not significant $(P=0.0885)$. As 15-HETE is produced by both COX activity (along with 11-HETE) and by $12 / 15$ LOX activity (along with 12-HETE) ${ }^{(43-46)}$, the levels of these combined HETE were also analysed. Both 11- and 15-HETE, as well as 12- and 15-HETE, were higher by 73 and $99 \%$, respectively, in MetS compared with lean rat kidneys (Fig. 3(g) and (h)). These combined levels were also reduced by the fish oil diet in MetS rats to levels similar to those in lean control rats. While the levels of other detectable HETE (5-, 8- and 9-HETE, Fig. 3(a)-(c)) in the kidney were not altered by genotype, fish oil treatment did reduce 5 - and 

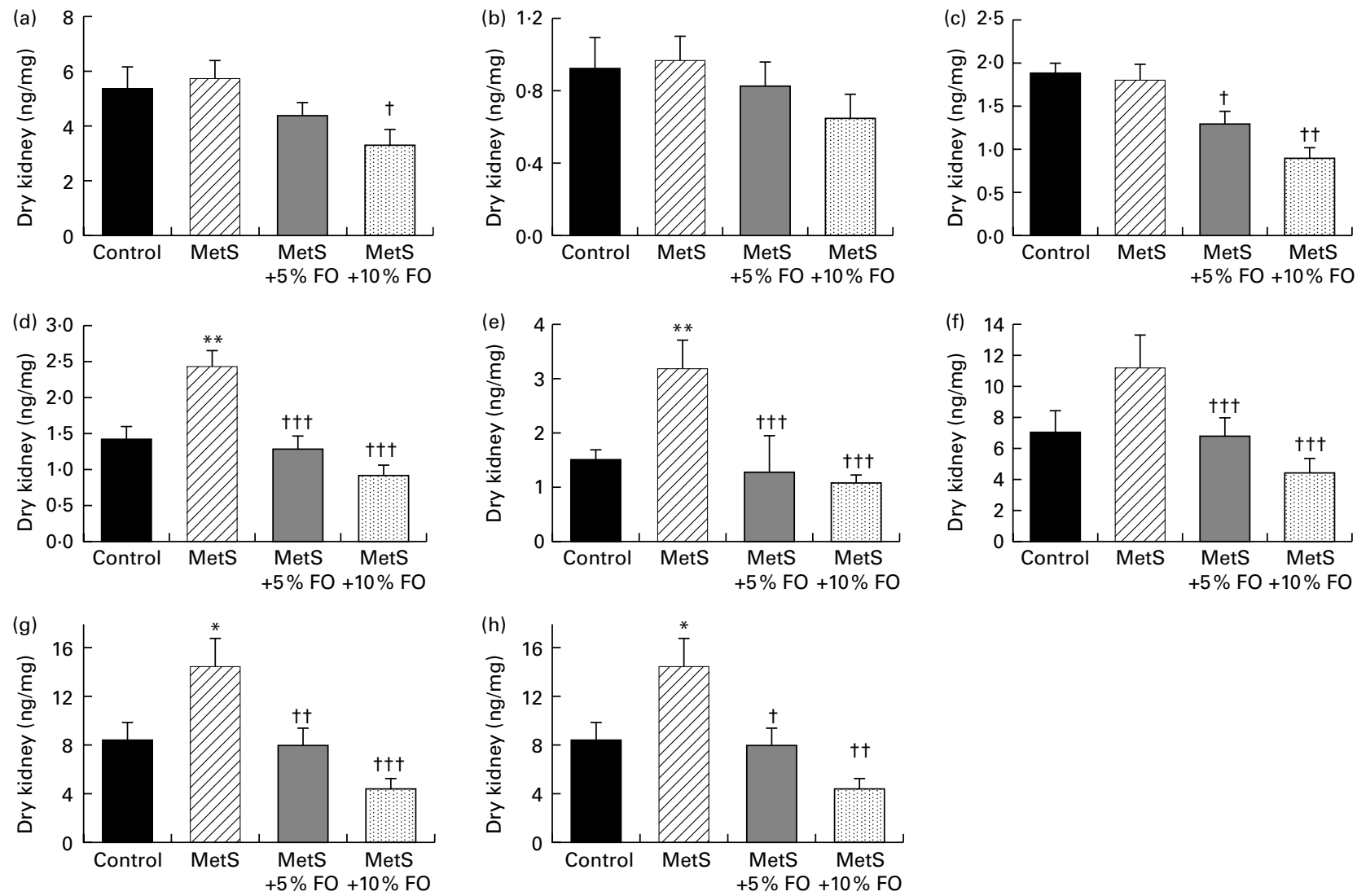

Fig. 3. Endogenous hydroxyeicosatetraenoic acid (HETE; (a) 5-HETE; (b) 8-HETE; (c) 9-HETE; (d) 11-HETE; (e) 12-HETE; (f) 15-HETE; (g) 11-HETE + $15-\mathrm{HETE}$; (h) 12-HETE + 15-HETE) levels in the kidneys of JCR:LA-cp rats in each of the treatment groups. Values are means, with standard errors represented by vertical bars $(n 8)$. Mean values were significantly different from that of the lean control: ${ }^{\star} P<0.05$, ${ }^{\star \star} P<0.01$. Mean values were significantly different from that of the metabolic syndrome (MetS) control: $\uparrow P<0.05$, $\dagger \uparrow P<0.01, \dagger \dagger \dagger P<0.001$. FO, fish oil.

9-HETE levels in MetS rats. Corresponding HEPE derived from EPA were not detected, nor was there significant production of HETE detected in vitro.

Endogenous prostanoids in kidneys were not different between untreated MetS and lean control rats (Table 3). However, fish oil feeding resulted in marked reductions of the 2 -series prostanoids. Of these prostanoids, 6-keto $\mathrm{PGF}_{1 \mathrm{a}}$, $\mathrm{TxB}_{2}, \mathrm{PGF}_{2 \alpha}$ and $\mathrm{PGD}_{2}$ were reduced by approximately $60 \%$ in the MetS rats fed the $10 \%$ fish oil diet compared with the untreated MetS rats. Kidneys from rats fed the 5\% fish oil had $40-50 \%$ lower levels of $\mathrm{TxB}_{2}$ and $\mathrm{PGF}_{2 \alpha}$ (Table 3) compared with the untreated MetS group. Interestingly, both fish oil diets resulted in the appearance of the 3-series prostanoids $\mathrm{PGE}_{3}$ and $\mathrm{PGD}_{3}$, which were not detectable in kidneys from untreated MetS rats.

When in vitro production of eicosanoids was examined, only prostanoids were produced and subsequently used to assess COX activity. Consistent with endogenous levels, in vitro production of renal prostanoids was not affected by genotype, but dietary fish oil markedly reduced COX activity in MetS rats in both the 5 and $10 \%$ fish oil groups, compared with the LBD group (Table 3). COX activity, as measured by four out of five prostanoids, was reduced by $63-99 \%$ in the $10 \%$ fish oil group, with lesser effects in the 5\% fish oil group. Overall, COX activity was reduced in kidneys by
$72 \%$ in the $5 \%$ fish oil group and by $78 \%$ in the $10 \%$ fish oil group, compared with the kidneys from untreated MetS rats (Table 3 ).

\section{Discussion}

The purpose of the present study was to examine whether a long-term fish oil-supplemented diet would improve renal function and glomerulosclerosis in the MetS JCR:LA- $c p$ rat model. A secondary objective was to investigate the corresponding renal eicosanoid profile and the effects of dietary fish oil on eicosanoid levels. The present findings show that chronic fish oil feeding can improve renal pathology and normalise the elevated HETE levels associated with the MetS in the JCR:LA- $c p$ rat.

Urinary albumin is an indicator of renal microvascular damage, and often reflects elevated glomerular permeability and inability to retain albumin ${ }^{(47)}$. Glomerulosclerosis is a major cause of end-stage renal failure in diabetic and obese individuals, impairing the filtering process and allowing protein to leak into the urine ${ }^{(48)}$. In the present study, JCR:LA- $c p$ rats fed fish oil had significantly less albuminuria, glomerulosclerosis and obesity. These results are consistent with previous studies of dietary fish oil in other models of renal disease. Diabetic KKAy/Ta mice injected 
Table 3. Endogenous prostanoid levels in the kidneys of lean and metabolic syndrome (MetS) JCR:LA-cp rats fed a lipid-balanced control diet (LBD), or a 5 or $10 \%$ fish oil (FO) diet

(Mean values with their standard errors, $n 8$ per group)

\begin{tabular}{|c|c|c|c|c|c|c|c|c|}
\hline \multirow{3}{*}{$\begin{array}{l}\text { Genotype... } \\
\text { Diet... }\end{array}$} & \multirow{2}{*}{\multicolumn{2}{|c|}{$\frac{\text { Control }}{\text { LBD }}$}} & \multicolumn{6}{|c|}{ MetS } \\
\hline & & & \multicolumn{2}{|c|}{ LBD } & \multicolumn{2}{|c|}{ MetS $+5 \%$ FO } & \multicolumn{2}{|c|}{ MetS $+10 \%$ FO } \\
\hline & Mean & SEM & Mean & SEM & Mean & SEM & Mean & SEM \\
\hline \multicolumn{9}{|c|}{ Endogenous levels (ng/mg dry kidney) } \\
\hline 6-keto-PGF ${ }_{1 \alpha}$ & 0.42 & 0.07 & 0.40 & 0.06 & 0.26 & 0.05 & 0.17 & $0.02^{\star \star}$ \\
\hline $\mathrm{TxB}_{2}$ & $0 \cdot 11$ & 0.01 & 0.14 & 0.02 & 0.07 & $0.01^{\star \star \star}$ & 0.05 & $0.01^{\star * *}$ \\
\hline $\mathrm{PGF}_{2 \alpha}$ & 0.28 & 0.03 & 0.21 & 0.03 & 0.12 & $0.02^{*}$ & 0.08 & $0.01^{* \star *}$ \\
\hline $\mathrm{PGE}_{2}$ & 0.19 & 0.03 & 0.21 & 0.04 & 0.20 & 0.05 & 0.14 & 0.05 \\
\hline $\mathrm{PGD}_{2}$ & 0.34 & 0.05 & 0.43 & 0.09 & 0.37 & 0.06 & 0.18 & $0.04^{\star \star}$ \\
\hline Total 2-series & 1.34 & 0.14 & 1.39 & 0.24 & 0.98 & 0.13 & 0.62 & $0 \cdot 10^{\star \star}$ \\
\hline \multicolumn{9}{|l|}{ Prostanoids } \\
\hline $\mathrm{PGE}_{3}$ & \multirow{2}{*}{\multicolumn{2}{|c|}{ ND }} & \multicolumn{2}{|c|}{ ND } & 0.08 & $0.03^{\star *}$ & 0.09 & $0.04^{\star *}$ \\
\hline $\mathrm{PGD}_{3}$ & & & \multicolumn{2}{|c|}{ ND } & 0.23 & $0.08^{* *}$ & 0.17 & $0.05^{\star *}$ \\
\hline Total prostanoids & $1 \cdot 34$ & 0.14 & $1 \cdot 39$ & 0.24 & 1.25 & 0.20 & 0.88 & 0.18 \\
\hline \multicolumn{9}{|c|}{ In vitro production (pg/mg dry kidney per min) } \\
\hline 6-keto-PGF ${ }_{1 \alpha}$ & $29 \cdot 1$ & 8.4 & $46 \cdot 7$ & $6 \cdot 5$ & $22 \cdot 8$ & $7 \cdot 3^{*}$ & 14.6 & $1.7^{\star \star}$ \\
\hline $\mathrm{TxB}_{2}$ & $6 \cdot 2$ & $2 \cdot 3$ & $6 \cdot 8$ & $1 \cdot 3$ & 4.6 & 1.5 & $1 \cdot 2$ & $0.7^{\star \star}$ \\
\hline $\mathrm{PGF}_{2 \alpha}$ & 45.4 & $6 \cdot 2$ & $39 \cdot 8$ & $6 \cdot 2$ & $8 \cdot 1$ & $2 \cdot 4^{\star \star *}$ & 9.5 & $1 \cdot 1^{\star \star *}$ \\
\hline $\mathrm{PGE}_{2}$ & $11 \cdot 7$ & $3 \cdot 0$ & $14 \cdot 8$ & $1 \cdot 1$ & 0.2 & $4 \cdot 5^{\star \star}$ & 0.1 & $2 \cdot 5^{\star \star}$ \\
\hline $\mathrm{PGD}_{2}$ & $30 \cdot 8$ & 14.9 & $34 \cdot 3$ & $12 \cdot 6$ & $10 \cdot 6$ & 9.9 & $15 \cdot 9$ & 3.8 \\
\hline Total activity & 143 & $30 \cdot 0$ & 159 & $22 \cdot 8$ & $51 \cdot 7$ & $23 \cdot 6^{\star \star \star}$ & $41 \cdot 3$ & $7 \cdot 9^{\star \star \star}$ \\
\hline
\end{tabular}

$\mathrm{TxB}_{2}$, thromboxane $\mathrm{B}_{2} ; \mathrm{ND}$, not determined.

Mean values were significantly different from that of the MetS control: ${ }^{*} P<0.05,{ }^{* \star} P<0.01,{ }^{* \star *} P<0.001$.

intraperitoneally with EPA ethyl ester ( $1 \mathrm{~g} / \mathrm{kg}$ per d) had attenuated glomerulosclerosis, mesangial matrix accumulation, tubulointerstitial inflammation and albuminuria ${ }^{(49)}$. In a model of focal glomerulosclerosis, a fish oil-enriched diet substantially lowered urine albumin excretion, probably reflecting a lower severity of kidney disease ${ }^{(50)}$. In human studies of diabetic nephropathy, reduced albuminuria is the most common, although not consistent, finding with fish oil supplementation $^{(37)}$. The beneficial effects of fish oil on renal injury in the JCR:LA-cp model may be due to the improvement in hyperlipidaemia and insulinaemia previously reported from this study ${ }^{(12)}$, as high circulating levels of lipids and insulin cause renal damage $\mathrm{e}^{(7,8,10,11)}$.

Dietary fish oil also could be nephroprotective via alterations in eicosanoid metabolism. Eicosanoids are biologically active molecules that play important and sometimes opposing roles in kidney function ${ }^{(15,27)} . \mathrm{PGD}_{2}, \mathrm{PGE}_{2}$ and HETE are generally associated with pro-inflammatory effects, but $\mathrm{PGE}_{2}$ as well as $\mathrm{PGI}_{2}$ also are vasodilators and are important in maintaining normal renal plasma flow ${ }^{(51,52)} \cdot \mathrm{PGE}_{2}$ is also involved in the regulation of $\mathrm{Na}$ re-absorption and renal cell over-proliferation and formation of fibrous tissue ${ }^{(14,53,54)}$. Thromboxane $\mathrm{A}_{2}$ and 12-HETE are known to be vasoconstrictors that are involved in the pathogenesis of hypertension associated with long-term renal failure ${ }^{(30,55)}$, as well as being elevated in models of diabetic nephropathy ${ }^{(21-23)}$

The present findings in the JCR:LA- $c p$ rat demonstrate that elevated renal HETE levels are associated with renal pathology in the MetS, supporting previous studies that indicated the importance of elevated HETE in other forms of renal injury $^{(21-23)}$. Several studies have shown that by blocking the levels of the 12/15 LOX enzyme using inhibitors or gene knockout strategies, glomerular volume, mesangial cell hypertrophy, extracellular matrix formation and proteinuria can be reduced in models of diabetic nephropathy ${ }^{(24-26,43)}$. The similar effect of fish oil on HETE levels in the JCR:LA-cp rat indicates that these eicosanoids may also be key mediators of renal pathology associated with the MetS

In contrast to the elevated HETE levels in MetS rat kidneys, renal prostanoid levels were not altered. This may have been due to the relatively early stage of renal injury present in this model, which would indicate that HETE alterations occur earlier in disease progression than perturbations in prostanoid levels. Nevertheless, dietary fish oil also reduced the endogenous levels and in vitro production of most prostanoids. The reduced levels of the 2-series prostanoids and HETE are consistent with previous reports that demonstrated that dietary $n-3$ PUFA can inhibit the production of eicosanoids derived from ARA. In these kidneys, the only eicosanoid products derived from $n-3$ PUFA detected were $\mathrm{PGD}_{3}$ and $\mathrm{PGE}_{3}$, demonstrating selectivity for the eicosanoids produced from EPA. Previous studies have shown that $n$-3 PUFA reduce the expression of COX messenger RNA and protein both in vivo and in vitro ${ }^{(44-46)} . n$-3 PUFA accumulate in the plasma membrane and partially replace ARA as substrates for COX and LOX, resulting in reduced products from ARA and enhanced production from $\mathrm{EPA}^{(56,57)}$. Both protective and detrimental 2 -series prostanoids were reduced in rats treated with fish oil, so their overall effect on the kidney is difficult to assess. However, the 3-series prostanoids tend to be much less bioactive ${ }^{(28-30)}$, so the reduction of ARA-derived products in combination with the enhanced EPA-derived eicosanoids would probably have decreased the overall bioactivity of the eicosanoids present in rats treated with fish oil. 
Caution regarding the use of high levels of fish oil for the treatment of kidney injury in the MetS is warranted, as the $10 \%$ fish oil diet resulted in higher levels of tubular damage. Few studies have reported adverse effects of high-dose fish oil on the kidney, but the BHE/Cdb rat model of diabetic nephropathy also had worsened glomerular histopathology and shortened lifespan when given $9 \%$ fish oil ${ }^{(58)}$. The $p c y$ mouse model of adolescent nephronophthisis also exhibits greater fibrosis and cyst growth when given high levels of dietary long-chain $n$-3 PUFA $^{(59,60)}$. It is possible that whilst attenuation of HETE, which were elevated in the kidneys of MetS rats, may reduce renal damage, the reduction of protective 2-series prostanoids in the rats provided 10\% fish oil could have had detrimental effects on the development of renal pathology. It is not clear why the detrimental effect of $10 \%$ fish oil is observed specifically with glomerulosclerosis and not with albuminuria, but may point to a greater sensitivity to lower prostanoid levels. Whilst treatment with drugs that inhibit prostanoid formation ameliorate kidney dysfunction in some models ${ }^{(61-63)}$, nephrotoxicity from prostanoid inhibitors has also been documented ${ }^{(15,64)}$. Hence, simultaneous analysis of both prostanoids and HETE is important in order to obtain a more comprehensive understanding of potential eicosanoid roles in renal injury.

\section{Concluding remarks}

The findings of the present study offer evidence of the importance of elevated HETE levels in renal injury in the MetS. Furthermore, supplementation of moderate levels of $n-3$ PUFA derived from fish oil in the JCR:LA- $c p$ MetS rat reduces disease progression. We propose that long-term dietary intake of fish oil may improve glomerulosclerosis and kidney dysfunction associated with elevated renal HETE levels in the MetS.

\section{Acknowledgements}

The research was supported, in part, by a pilot grant from the Alberta Diabetes Institute (S. D. P.) and NSERC Discovery grants (S. D. P. and H. M. A.). S. D. P. is a HSFC New Investigator. The authors wish to declare no conflict of interest. We wish to thank Kristina MacNaughton and Sharon Sokolik for their excellent technical assistance throughout the present study. Thank you to Jennifer Wizbicki, Joy Gauthier and Tanja Winter for technical assistance with the eicosanoid analysis. S. D. P., J. L. and H. M. A. conceived and designed the experiments. J. L. performed the experiments. The data were analysed by J. L., S. D. P., H. M. A. and F. B. The manuscript was prepared by H. M. A., S. D. P. and F. B.

\section{References}

1. Russell JC, Graham SE \& Richardson M (1998) Cardiovascular disease in the JCR:LA-cp rat. Mol Cell Biochem 188, 113-126.

2. Russell JC, Graham SE \& Hameed M (1994) Abnormal insulin and glucose metabolism in the JCR:LA-corpulent rat. Metabolism 43, 538-543.
3. Russell JC, Amy RM, Graham SE, et al. (1995) Inhibition of atherosclerosis and myocardial lesions in the JCR:LA-cp rat by beta, beta'-tetramethylhexadecanedioic acid (MEDICA 16). Atheroscler Thromb Vasc Biol 15, 918-923.

4. Mangat R, Su J, Scott PG, et al. (2007) Chylomicron and apoB48 metabolism in the JCR:LA- $C P$ corpulent rat, a model for the metabolic syndrome. Biochem Soc Trans 35, 477-481

5. Proctor SD, Kelly SE, Stanhope KL, et al. (2007) Synergistic effects of conjugated linoleic acid and chromium picolinate improve vascular function and renal pathophysiology in the insulin-resistant JCR:LA-cp rat. Diabetes Obes Metab 9 , $87-95$.

6. Thomas G, Sehgal AR, Kashyap SR, et al. (2011) Metabolic syndrome and kidney disease: a systematic review and meta-analysis. Clin J Am Soc Nephrol 6, 2364-2373.

7. Abrass CK (2004) Cellular lipid metabolism and the role of lipids in progressive renal disease. Am J Nephrol 24, 46-53

8. Chen J, Munter P, Hamm LL, et al. (2003) Insulin resistance and risk of coronary kidney disease in nondiabetic US adults. J Am Soc Nephrol 14, 469-477.

9. Chen J, Wildman RP \& Hamm L (2004) Association between inflammation and insulin resistance in U.S. nondiabetic adults: results from the third National Health and Nutrition Examination Survey. Diabetes Care 27, 2960-2965.

10. Wang Z, Jiang T, Li J, et al. (2005) Regulation of renal lipid metabolism, lipid accumulation, and glomerulosclerosis in $\mathrm{fvbdb} / \mathrm{db}$ mice with type 2 diabetes. Diabetes $\mathbf{5 4}$, $2328-2335$.

11. Schaffer JE (2003) Lipotoxicity: when tissues overeat. Curr Opin Lipidol 14, 281-287.

12. Lu J, Borthwick F, Hassanali Z, et al. (2011) Chronic dietary $n$-3 PUFA intervention improves dyslipidemia and subsequent cardiovascular complications in the JCR:LA-cp rat model of the metabolic syndrome. Br J Nutr 105, $1572-1582$.

13. Klahr S (1989) Intervention studies in diabetic nephropathy. Contrib Nephrol 73, 147-158.

14. Remuzzi G, Ruggeneti P \& Benigni A (1997) Understanding the nature of renal disease progression. Kidney Int 51, 2-15.

15. Hao CM \& Breyer MD (2008) Physiological regulation of prostaglandins in the kidney. Annu Rev Physiol 70, 357-377.

16. Schambelan M, Blake S, Sraer J, et al. (1985) Increased prostaglandin production by glomeruli isolated from rats with streptozotocin-induced diabetes mellitus. J Clin Invest 75, 404-412.

17. Komers R, Zdychová J, Cahová M, et al. (2005) Renal cyclooxygenase-2 in obese Zucker (fatty) rats. Kidney Int 67, 2151-2158.

18. Komers R, Lindsley J, Oyama T, et al. (2007) Cyclo-oxygenase-2 inhibition attenuates the progression of nephropathy in uninephrectomized diabetic rats. Clin Exp Pharmacol Physiol 34, 36-41.

19. Craven PA, Caines MA \& DeRubertis FR (1987) Sequential alterations in glomerular prostaglandin and thromboxane synthesis in diabetic rats: relationship to the hyperfiltration of early diabetes. Metabolism 36, 95-103.

20. Hao CM \& Breyer MD (2007) Physiologic and pathophysiologic roles of lipid mediators in the kidney. Kidney Int $\mathbf{7 1}$, 1105-1115.

21. Antonipillai I, Nadler J, Vu EJ, et al. (1996) A 12-lipoxygenase product, 12-hydroxyeicosatetraenoic acid is increased in diabetics with incipient and early renal disease. $J$ Clin Endocrinol Metab 81, 1940-1945.

22. Kang SW, Adler SG, Nast CC, et al. (2001) 12-Lipoxygenase is increased in glucose-stimulated mesangial cells and 
in experimental diabetic nephropathy. Kidney Int $\mathbf{5 9}$ $1354-1362$.

23. Xu ZG, Miao LN, Cui YC, et al. (2009) Angiotensin II type 1 receptor expression is increased via 12-lipoxygenase in high glucose-stimulated glomerular cells and type 2 diabetic glomeruli. Nephrol Dial Transplant 24, 1744-1752.

24. Kim YS, Reddy MA, Lanting L, et al. (2003) Differential behavior of mesangial cells derived from 12/15 lipoxygenase knockout mice relative to control mice. Kidney Int $\mathbf{6 4}$, 1702-1714.

25. Anning PB, Coles B, Bermudez-Fajardo A, et al. (2005) Elevated endothelial nitric oxide bioactivity and resistance to angiotensin-dependent hypertension in 12/15-lipooxygenase knockout mice. Am J Pathol 166, 653-662.

26. Guo OY, Mia LN, Li B, et al. (2011) Role of 12-lipoxygenase in decreasing $P$-adherin and increasing angiotensin II type 1 receptor expression according to glomerular size in type 2 diabetic rats. Am J Physiol Endocrinol Metab 300, E708-E716.

27. Câmara NO, Martins JO \& Landgraf RG (2009) Emerging roles of eicosanoids in renal diseases. Curr Opin Nephrol Hypertens 18, 21-27.

28. Grimminger K, Mayer K, Kramer HJ, et al. (1993) Differential vasoconstrictor potencies of free fatty acids in the lung vasculature: 2- versus 3-series prostanoid generation. J Pharmacol Exp Ther 267, 259-265.

29. Kulkarni PS \& Srinivasan BD (1986) Eicosapentaenoic acid metabolism in human and rabbit anterior urea. Prostaglandins 31, 1159-1164.

30. Darlametsos IE \& Varnos DD (2001) Role of prostanoids and endothelins in the prevention of cyclosporine-induced nephrotixicity. Prostaglandins Leukot Essent Fatty Acids 64 , 231-239.

31. Donadio JV \& Grande JP (2004) The role of fish oil/omega-3 fatty acids in the treatment of IgA nephropathy. Semin Nephrol 24, 225-243.

32. Koop K, Eikmans M, Wwehland M, et al. (2008) Selective loss of podoplanin protein expression accompanies proteinuria and precedes alterations in podocyte morphology in a spontaneous proteinuric rat model. Am J Pathol 173, 315-326.

33. Lu J, Bankovic-Calic N \& Ogborn M (2003) Detrimental effects of high fat diet in early renal injury are ameliorated by fish oil in Han:SPRD-cyc rats. J Nutr 133, 180-186.

34. Sinha AK, Scharschmidt LA, Neuwirth R, et al. (1990) Effects of fish oil on glomerular function in rats with diabetes mellitus. J Lipid Res 31, 1219-1228.

35. Weise WJ, Natori Y, Levine JS, et al. (1993) Fish oil has protective and therapeutic effects on proteinuria in passive Heymann nephritis. Kidney Int 43, 359-368.

36. Fassett RG, Gobe GC, Peake JM, et al. (2010) Omega-3 polyunsaturated fatty acids in the treatment of kidney disease. Am J Kidney Dis 56, 728-742.

37. Shapiro H, Theilla M, Attal-Singer J, et al. (2011) Effects of polyunsaturated fatty acid consumption in diabetic nephropathy. Nat Rev Nephrol 7, 110-121.

38. Carfray A, Patel K, Whitaker P, et al. (2000) Albumin as an outcome measure in haemodialysis in patients: the effect of variation in assay method. Nephrol Dial Transplant 15, 1819-1822.

39. Heidbreder E, Schafferhans K, Schramm D, et al. (1986) Toxic renal failure in the rat: beneficial effects of atrial natriuretic factor. Klin Wochenschr 65, 78-82.

40. Warford-Woolgar L, Peng CY, Shuhyta J, et al. (2006) Selectivity of cyclooxygenase isoform activity and prostanoid production in normal and diseased Han:SPRD-cy rat kidneys. Am J Physiol Renal Physiol 290, F897-F904.

41. Deems R, Buczynski MW, Bowers-Gentry R, et al. (2007) Detection and quantitation of eicosanoids via high-performance liquid chromatography-electrospray ionization-mass spectrometry. Methods Enzymol 432, 59-82.

42. Hall LM \& Murphy RC (1998) Electrospray mass spectrometric analysis of 5-hydropeoxy and 5-hydroxyeicosatetraenoic acids generated by lipid peroxidation of red blood cells ghost phospholipids. J Am Soc Mass Spectrom 9, 527-532.

43. Dobrian AD, Lieb AC, Cole BK, et al. (2011) Functional and pathological roles of the 12- and 15-lipoxygenases. Prog Lipid Res 50, 115-131.

44. Mund RC, Pizato N, Bonatto S, et al. (2007) Decreased tumor growth in walker 256 tumor-bearing rats chronically supplemented with fish oil involves COX-2 and PGE2 reduction associated with apoptosis and increased peroxidation. Prostaglandins Leukot Essent Fatty Acids 76, $113-120$.

45. Sankaran D, Lu J, Ogborn MR, et al. (2007) COX-2 expression in cystic kidneys is dependent on dietary $n$-3 fatty acid composition. $J$ Nutr Biochem 18, 806-812.

46. Calviello G, Di Nicuolo F, Gragnoli S, et al. (2004) n-3 PUFAs reduce VEGF expression in human colon cancer cells modulating the COX-2/PGE2 induced ERK-1 and -2 and HIF-1alpha induction pathway. Carcinogenesis 25, 23032310 .

47. Hamano K, Nitta A, Ohtake T, et al. (2008) Associations of renal vascular resistance with albuminuria and other macroangiopathy in type 2 diabetic patients. Diabetes Care 31, 1853-1857.

48. Eknoyan G (2007) Obesity, diabetes, and chronic kidney disease. Curr Diab Rep 7, 449-453.

49. Hagiwara S, Makita Y, Gu L, et al. (2006) Eicosapentaenoic acid ameliorates diabetic nephropathy of type 2 diabetic KKAy/Ta mice: involvement of MCP-1 suppression and decreased ERK1/2 and p38 phosphorylation. Nephrol Dial Transplant 21, 605-615.

50. Goldstein DJ, Wheeler DC, Sandstorm DJ, et al. (1995) Fish oil ameliorates renal injury and hyperlipidemia in the Milan normotensive rat model of focal glomerulosclerosis. J Am Soc Nephrol 6, 1468-1475.

51. Kappor M, Kojima F, Yang L, et al. (2007) Sequential induction of pro- and anti-inflammatory prostaglandins and peroxisome proliferators-activated receptor-gamma during normal wound healing: a time course study. Prostaglandins Leukot Essent Fatty Acids 76, 103-112.

52. Mori A, Saito M, Sakamoto K, et al. (2007) Stimulation of prostanoid IP and EP(2) receptors dilates retinal arterioles and increases retinal and choroidal blood flow in rats. Eur J Pharmacol 570, 135-141.

53. Hodeify RF \& Kreydiyyeh SI (2007) PGE2 reduces net water and chloride absorption from the rat colon by targeting the $\mathrm{Na}^{+} / \mathrm{H}^{+}$exchanger and the $\mathrm{Na}^{+} \mathrm{K}^{+} 2 \mathrm{Cl}^{-}$cotransporter. Prostaglandins Leukot Essent Fatty Acids 76, 285-292.

54. Kitahara M, Eitner F, Ostendorf T, et al. (2002) Selective cyclooxygenase-2 inhibition impairs glomerular capillary healing in experimental glomerulonephritis. I Am Soc Nephrol 13, 1261-1270.

55. Larivière R, Moreau C, Rodrigue ME, et al. (2004) Thromboxane blockade reduces blood pressure and progression of renal failure independent of endothelin-1 in uremic rats. Prosatglandins Leukot Essent Fatty Acids 71, 103-109.

56. Smith WL (2005) Cyclooxygenases, peroxide tone and the allure of fish oil. Curr Opin Cell Biol 17, 174-182. 
57. Needleman P, Wyche A \& Bronson SD (1979) Specific regulation of peptide-induced renal prostaglandin synthesis. J Biol Chem 254, 9772-9779.

58. Berdanier CD, Johnson B, Hartle DK, et al. (1992) Life span is shortened in BHE/cdb rats fed a diet containing $9 \%$ menhaden oil and $1 \%$ corn oil. J Nutr 122, $1309-1317$

59. Sankaran D, Lu J, Bankovich-Calic N, et al. (2004) Modulation of renal injury in $p c y$ mice by dietary fat containing $(n-3)$ fatty acids depends on age at intervention, level of fat and type of (n-3) fatty acid. Lipids 39, 207-214.

60. Aukema HM, Yamaguchi T, Takahashi H, et al. (1992) Effects of dietary fish oil on survival and renal fatty acid composition in murine polycystic kidney disease. Nutr Res 1383-1392.
61. Norby LH, Wweidig J, Ramwell P, et al. (1978) Possible role for impaired renal prostaglandin in pathogenesis if hyporeninaemic hypoaldosteronism. Lancet ii, 1118-1122.

62. Brater DC, Harris C, Redfern JS, et al. (2001) Renal effects of COX-2-selective inhibitors. Am J Nephrol 21, 1-15.

63. Arisz L, Donker AJ, Brentjens JR, et al. (1976) The effect of indomethacin on proteinuria and kidney function in the nephritic syndrome. Acta Med Scand 199, 121-125.

64. Sankaran D, Bankovich-Calic N, Crow G, et al. (2007) Selective COX-2 inhibition markedly slows disease progression and attenuates altered prostanoid production in Han:SPRDcy rats with inherited kidney disease. Am J Physiol Renal Physiol 293, F821-F830. 